\title{
Novel Bayesian Approach to Assess System Availability using a Threshold to Censor Data
}

\author{
Esi Saari ${ }^{\mathrm{a}}$, Jing Lin ${ }^{\mathrm{a}, *}$, Bin Liu ${ }^{\mathrm{b}}$, Liangwei Zhang ${ }^{\mathrm{a}, \mathrm{c}}$, and Ramin Karim ${ }^{\mathrm{a}}$ \\ ${ }^{a}$ Division of Operation and Maintenance Engineering, Luleå University of Technology, Luleå, 97897, Sweden \\ ${ }^{b}$ Department of Management Science, University of Strathclyde, Glasgow, G1 1XQ, UK \\ ${ }^{c}$ Department of Industrial Engineering, Dongguan University of Technology, Dongguan, 523808, China
}

\begin{abstract}
Assessment of system availability has been studied from the design stage to the operational stage in various system configurations using either analytic or simulation techniques. However, the former cannot handle complicated state changes, and the latter is computationally expensive. This study proposes a Bayesian approach to evaluate system availability. In this approach: 1) Mean Time to Failure (MTTF) and Mean Time to Repair (MTTR) are treated as distributions instead of being "averaged" to better describe real scenarios and overcome the limitations of data sample size; 2) Markov Chain Monte Carlo (MCMC) simulations are applied to take advantage of the analytical and simulation methods; and 3) a threshold is set up for Time to Failure (TTR) data and Time to Repair (TTR) data, and new datasets with right-censored data are created to reveal the connections between technical and "Soft" KPIs. To demonstrate the approach, the paper considers a case study of a balling drum system in a mining company. In this system, MTTF and MTTR are determined by a Bayesian Weibull model and a Bayesian lognormal model, respectively. The results show that the proposed approach can integrate the analytical and simulation methods to assess system availability and could be applied to other technical problems in asset management (e.g., other industries, other systems). By comparing the results with and without considering the threshold for censoring data, we show the threshold can be used as a monitoring line for continuous improvement in the investigated mining company.
\end{abstract}

Keywords: system availability; Bayesian statistics; Gibbs sampling; Kaplan-Meier estimation; mining industry

(Submitted on February 22, 2019; Revised on April 20, 2019; Accepted on May 18, 2019)

(C) 2019 Totem Publisher, Inc. All rights reserved.

\section{Introduction}

Availability, commonly measured as Mean Time to Failure (MTTF) and Mean Time to Repair (MTTR), is one of the most critical aspects of performance evaluation. Approaches to assessing system availability mainly use either analytic or simulation techniques (note: PC tools and databases are other options, but they are not part of this research).

Simulation techniques estimate availability by simulating the actual process and random behavior of the system. The advantage is that non-Markov failures and repair processes can be modeled easily [1-4], as can multi-state systems with operational dependencies [5]. Although simulation is more flexible, it is computationally expensive. In general, analytic techniques represent systems that use mathematical solutions from applied probability theory to make statements on various performance measures [2, 6-8]. However, such approaches have been criticized as too restrictive to tackle practical problems; they assume constant failure and repair rates, and this is not likely to be the case in the real world. Furthermore, the time-dependent availability obtained by a Markovian assumption (a common analytic technique) is not valid for nonMarkovian processes [1]. Traditionally, Bayesian statistical approaches have been used to assess system availability as they can solve the problem of complicated system state changes and computationally expensive simulation data, but they require strict assumptions on prior forms and can be computationally difficult. Bayesian research is more concerned with the prior's selection or the posterior's computation than the reality [9-13].

This study proposes a novel Bayesian approach to system availability assessment, combining analytic and simulation

\footnotetext{
* Corresponding author.

E-mail address: janet.lin@1tu.se
} 
techniques. In the proposed approach: 1) Mean Time to Failure (MTTF) and Mean Time to Repair (MTTR) are treated as distributions instead of being "averaged" to better reflect reality and compensate for the limitations of simulation data sample size; 2) Markov Chain Monte Carlo (MCMC) simulations are used to take advantage of both analytical and simulation methods [14]; and 3) a threshold is established for Time to Failure (TTF) data and Time to Repair (TTR) data, and new datasets created with right-censored data reveal the connections between technical and "soft" KPIs.

The rest of this paper is organized as follows. Section 2 explains the three stages of the proposed Bayesian approach. Section 3 describes Stage I, the pre-analysis, including the configuration of a balling drum system in a case study mine, data collection and preparation, and the preliminary analysis of failure and repair data. Section 4 presents Stage II; it proposes a Bayesian Weibull model for MTTF and a Bayesian lognormal model for MTTR considering right-censored data and explains how to use an MCMC computational scheme to obtain the posterior distributions. Section 5 explains Stage III, the assessment of system availability. Section 6 presents and assesses the results of a case study and then compares results with and without considering the data censored by the threshold. Section 7 features a discussion, while Section 8 provides conclusions and makes suggestions for further study.

\section{A General Procedure}

The proposed Bayesian approach to system availability has seven steps divided into three stages (see Table 1): 1) in Stage I, we perform pre-analysis; 2) in Stage II, we create the analytic models (Bayesian) and simulation models (MCMC); 3) in Stage III, we assess system availability.

Table 1. A general procedure

\begin{tabular}{|c|c|l|l|}
\hline Stages & Steps & \multicolumn{1}{|c|}{ Name } & \multicolumn{1}{c|}{ Description } \\
\hline \multirow{3}{*}{ I } & 1 & $\begin{array}{l}\text { Configuration } \\
\text { determination }\end{array}$ & Determine dependencies among units and system configuration. \\
\cline { 2 - 4 } & 2 & Data collection & Collect prior information and event data, including reliability and maintenance data. \\
\cline { 2 - 5 } & 3 & Data preparation & $\begin{array}{l}\text { Clean data and remove outliers as needed. } \\
\text { Set up a threshold for censored data. }\end{array}$ \\
\cline { 2 - 5 } & 4 & $\begin{array}{l}\text { Preliminary } \\
\text { Analysis }\end{array}$ & Determine the distribution of prior information, TTF, and TTR for the Bayesian analytics in step 5. \\
\hline \multirow{3}{*}{ II } & 5 & $\begin{array}{l}\text { Bayesian analytic } \\
\text { modeling }\end{array}$ & According to steps 3 and 4, determine the likelihood function and Bayesian analytic models. \\
\cline { 2 - 5 } & 6 & MCMC simulation & $\begin{array}{l}\text { Define burn-in defined and implement MCMC simulation; perform convergence diagnostics and check Monte } \\
\text { Carlo error to confirm the effectiveness of the results. If not passed, go back to steps 4 and 5; if passed, go to } \\
\text { step 7. }\end{array}$ \\
\hline III & 7 & Assessment & $\begin{array}{l}\text { According to the simulation results for Bayesian analytic models and system configuration, determine } \\
\text { distributions of TTF and TTR and assess system availability. } \\
\text { Assessment could start with the prior information collection in step 2 for the next calculation period. }\end{array}$ \\
\hline
\end{tabular}

The seven steps follow a "PDCA" cycle; those in Stage I can be treated as the Plan stage, Stage II as the Do and Check stage, and Stage III as the Action stage. The outputs from Stage III could become input for Stage I for the next calculation period, so the results can be continuously improved.

To accomplish step 2, prior information can come from: 1) engineering design data, 2) component test data, 3) system test data, 4) operational data from similar systems, 5) field data in various environments, 6) computer simulations, 7) related standards and operation manuals, 8) experience data from similar systems, and 9) expert judgment and personal experience. Of these, the first seven yield objective prior data, and the last two provide subjective prior data. Prior data also take a variety of forms, including reliability data, the distribution of reliability parameters, moments, confidence intervals, quantiles, upper and lower limits, etc.

In step 3, a threshold is set up according to the asset management goals connected with the organization's business goals (see later sections for a more detailed discussion). In step 4, various types of priors can be used because of the flexibility of MCMC. In this study, since the balling drums in the case study mine are quite new, we adopt vague priors. In step 5, the likelihood function can differ according to the types of censored/truncated data, while the Bayesian analytics could differ according to the preliminary study of the baseline analysis of TTF and TTR. In step 6, checking the MCMC simulation can follow [14]. In step 7, system availability can also be described by an empirical distribution instead of an analytical one. 


\section{Stage I: Pre-Analysis}

\subsection{Configuration of Balling Drum System}

The case study mine has five balling drums, labeled 1-5. All five balling drums receive their feed for production in the same manner, and each balling drum is expected to produce the same amount of pellets at its maximum. According to the company, the balling drums are independent; if one breaks down, it does not affect the rest. As Figure 1 shows, the five balling drums are in parallel configuration.

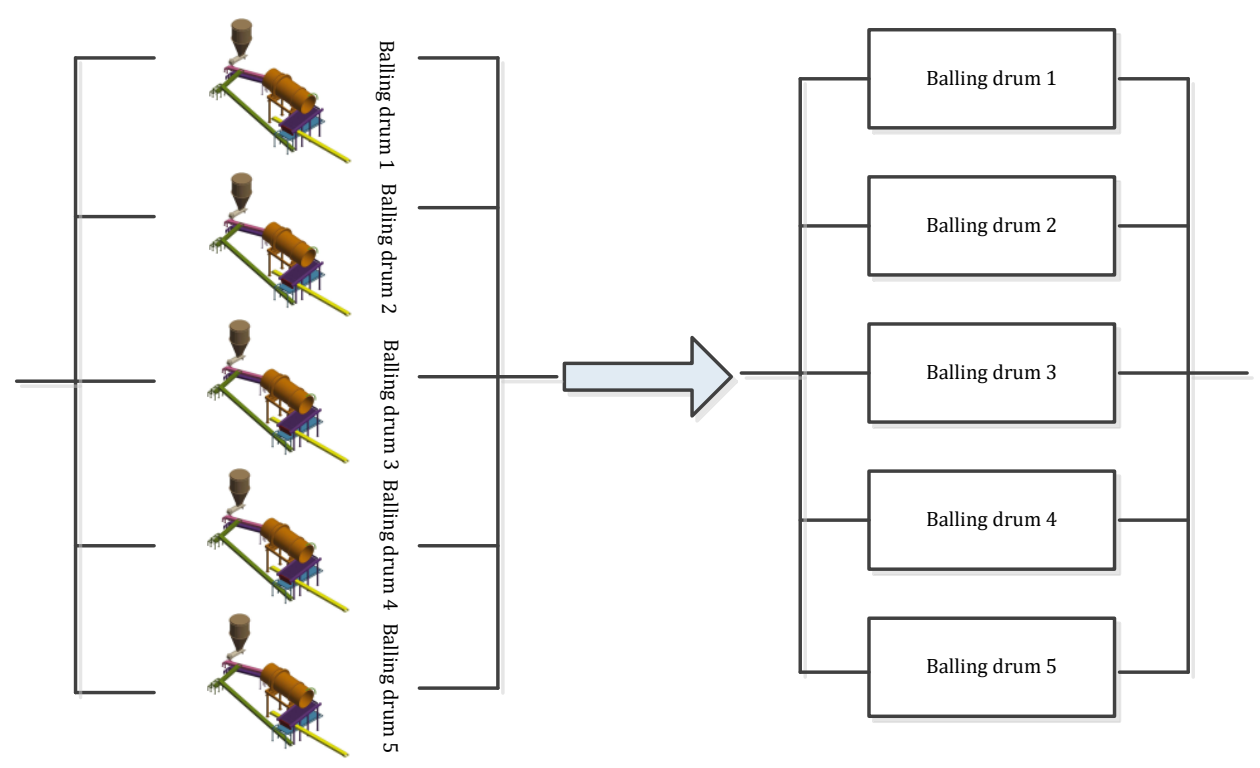

Figure 1. Description of a balling drum and the system sketch

The availability of a single balling drum, denoted as $A$, can be computed by

$$
A=\frac{M T T F}{M T T F+M T T R}
$$

The total system availability for this parallel configuration, $A_{\text {system }}$, can be calculated as

$$
A_{\text {system }}=1-\prod_{i=1}^{5}\left(1-A_{i}\right)
$$

\subsection{Data Collection and Data Preparation}

The study uses the failure and repair data of the five balling drums from January 2013 to December 2018 . There are 1,782 records. In the first step of data preparation, the null values are removed, and the data are reduced to 1,774 records.

In the next step, we look for the normal and abnormal values for the TTF and TTR of individual balling drums. If 150 shutdowns are considered normal, for example, then those exceeding 150 are abnormal, and 150 is denoted as a threshold, as shown in Figure 2. The work orders show that most of these abnormal shutdowns are caused by "preventive maintenance" and may simply reflect a lack of maintenance resources. To simplify the study, we assume that not all maintenance resources are sufficient for "preventive maintenance"; thus, the abnormal data may reflect a shortage of spare parts or skilled personnel.

To establish a more reasonable TTR threshold than the 150 shutdowns, we perform a Pareto analysis for all balling drums. The results appear in Figure 3. According to the figure, if the threshold is set up according to the "80-20" rule, the 
data can be censored at six hours. This explains almost $80 \%$ of the data. Therefore, we create a new dataset with TTR censored at six hours.

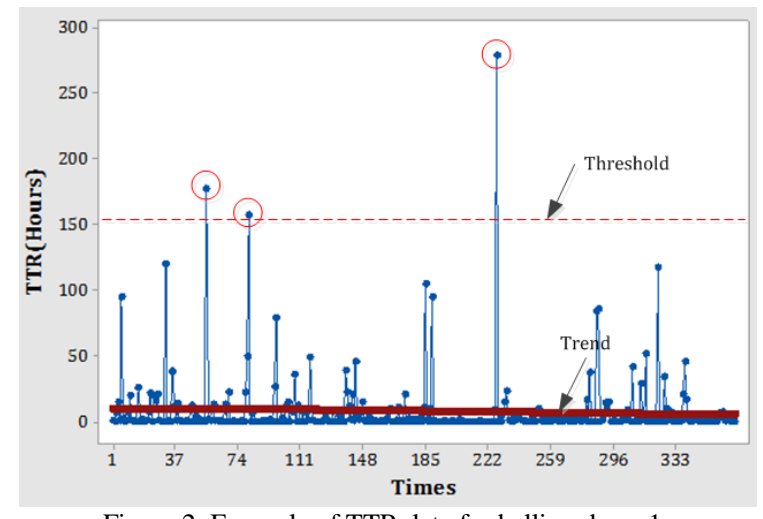

Figure 2. Example of TTR data for balling drum 1

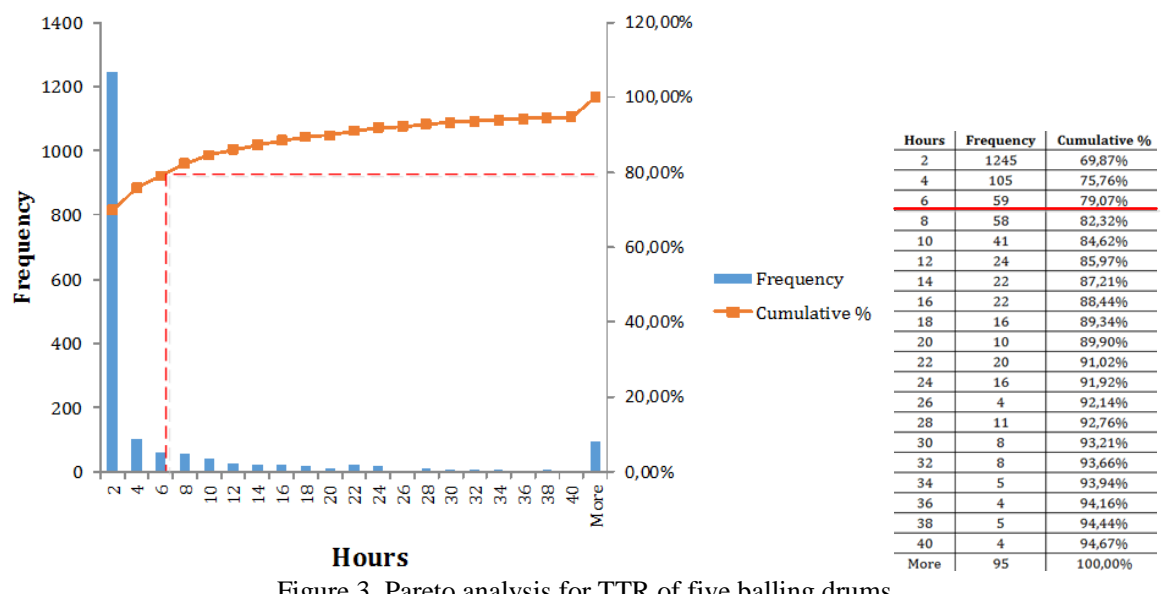

Figure 3. Pareto analysis for TTR of five balling drums

- Abnormal TTR values exceeding six hours could be improved by implementing maintenance improvements, including RCA, maintenance resource improvement, etc. The goal is to reduce the TTR values exceeding six hours. However, we do not know how much we can do. Therefore, those values are considered right-censored at six;

- The preventive maintenance plan is not changed. Thus, if one TTR is treated as censored, then in the corresponding maintenance interval, the Time Between Failure (TBF), which equals TTF plus TTR, will not change significantly, and the TTF could be longer than in the collected data. However, we do not know how much longer the TTF could be. Therefore, TTF data can also be treated as right-censored. The difference with censored TTR data is that the corresponding TTF data are treated as right-censored at the original value instead of a new value (see Figure 4).

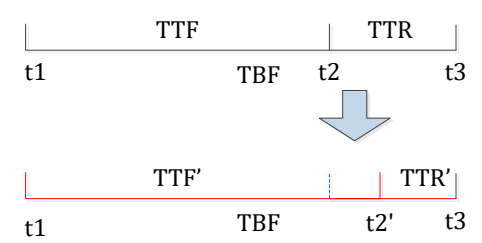

Figure 4. Data censored under assumptions

We use Figure 4 to illustrate assumption 2. TBF equals the time between $t_{1}$ and $t_{3}$. TTR $=t_{3}-t_{2}$ may be larger than six but it is right-censored at six. Then, the original TTR is denoted as six with a right-censored indicator. Since $T B F=t_{3}-$ $t_{1}$ will not change, the corresponding $T T F^{\prime}=t_{2}^{\prime}-t_{1}$ will be longer than TTF. However, according to assumption 2, we do not know how much longer; therefore, $T T F^{\prime}$ is denoted as right-censored data with an original value equal to $t_{2}-t_{1}$. After this step, the censored TTF and TTR data represent a total of $20 \%$ of all data. 


\subsection{Preliminary Analysis}

To determine the baseline distribution of $T T R$ and $T T F$, we conduct a preliminary study of failure data and repair data using traditional analysis. We consider the following distributions: exponential distribution, Weibull distribution, normal distribution, log-logistic distribution, lognormal distribution, and extreme value distribution. Table 2 lists the results, including the goodness-of-fit using Anderson-Darling (AD) statistics.

Table 2. Preliminary studies of failure data and repair data

\begin{tabular}{|c|c|c|c|c|c|c|c|c|}
\hline \multirow{2}{*}{$\begin{array}{c}\text { Balling } \\
\text { drum }\end{array}$} & \multicolumn{4}{|c|}{$T T F$ fitness } & \multicolumn{4}{|c|}{$T T R$ fitness } \\
\hline & $1 \mathrm{st}$ & $\mathrm{AD}$ & $2 \mathrm{nd}$ & $\mathrm{AD}$ & $1 \mathrm{st}$ & $\mathrm{AD}$ & 2nd & $\mathrm{AD}$ \\
\hline 1 & Weibull & 1.976 & Lognormal & 11.276 & Lognormal & 10.068 & Weibull & 14.607 \\
\hline 2 & Weibull & 1.796 & Lognormal & 8.274 & Lognormal & 11.144 & Weibull & 14.302 \\
\hline 3 & Weibull & 2.115 & Lognormal & 10.499 & Lognormal & 8.698 & Weibull & 14.332 \\
\hline 4 & Weibull & 1.196 & Lognormal & 6.366 & Lognormal & 9.245 & Weibull & 13.106 \\
\hline 5 & Weibull & 2.148 & Lognormal & 14.416 & Lognormal & 7.533 & Weibull & 11.933 \\
\hline
\end{tabular}

Based on the results, we select the Weibull distribution for the TTF and the lognormal distribution for the TTR and apply these to their respective parametric Bayesian models with censored data, as explained in the next section.

\section{Stage II: Analytic and Simulation Models}

This section elaborates on the analytic and simulation models described in Stage II. It proposes a Bayesian Weibull model for TTF and a Bayesian lognormal model for TTR and explains how to use an MCMC computational scheme to obtain the posterior distributions considering right-censored data.

\subsection{Markov Chain Monte Carlo with Gibbs Sampling}

The recent proliferation of Markov Chain Monte Carlo (MCMC) approaches has led to the use of the Bayesian inference in a wide variety of fields. MCMC is essentially Monte Carlo integration using Markov chains. Monte Carlo integration draws samples from the required distribution and then forms sample averages to approximate expected results. MCMC draws out these samples by running a cleverly constructed Markov chain for a long time. There are many ways to construct these chains. The Gibbs sampler is one of the best-known MCMC sampling algorithms in the Bayesian computational literature. In this method, when a set of parameters must be evaluated, the other parameters are assumed to be fixed and known. Let $\theta_{i}$ be an $i$-dimensional vector of parameters, and let $f\left(\theta_{j}\right)$ denote the marginal distribution for the $j^{\text {th }}$ parameter. The basic scheme of the Gibbs sampler for sampling from $p(\theta)$ comprises the following steps:

Step 1 Choose an arbitrary starting point $\theta^{(0)}=\left(\theta_{1}^{(0)}, \cdots, \theta_{k}{ }^{(0)}\right)$.

Step 2 Generate $\theta_{1}^{(1)}$ from the conditional distribution $f\left(\theta_{1} \mid \theta_{2}{ }^{(0)}, \cdots, \theta_{k}{ }^{(0)}\right)$, and generate $\theta_{2}^{(1)}$ from the conditional distribution $f\left(\theta_{2} \mid \theta_{1}^{(1)}, \theta_{3}{ }^{(0)}, \cdots, \theta_{k}{ }^{(0)}\right)$.

Step 3 Generate $\theta_{j}^{(1)}$ from $f\left(\theta_{j} \mid \theta_{1}^{(1)}, \cdots, \theta_{j-1}{ }^{(1)}, \theta_{j+1}{ }^{(1)}, \cdots, \theta_{k}{ }^{(0)}\right)$.

Step 4 Generate $\theta_{k}^{(1)}$ from $f\left(\theta_{k} \mid \theta_{1}^{(1)}, \theta_{2}{ }^{(1)}, \cdots, \theta_{k-1}{ }^{(1)}\right)$; the one-step transition from $\theta^{(0)}$ to $\theta^{(1)}=\left(\theta_{1}^{(1)}, \cdots, \theta_{k}^{(1)}\right)$ has been now completed, where $\theta^{(1)}$ is a one-time accomplishment of a Markov chain.

Step 5 Go to Step 2.

\subsection{Likelihood Construction for Right-Censored Data}

In practice, lifetime data are usually incomplete, and only a portion of the individual lifetimes of assets are known. Rightcensored data are often called Type I censoring in literature; the corresponding likelihood construction problem has been extensively studied. The right-censored data of this study are illustrated in Figure 4.

Suppose there are $n$ individuals whose lifetimes and censoring times are independent. The $\mathrm{i}$ th individual has life time $T_{i}$ and censoring time $L_{i}$. The $T_{i} \mathrm{~s}$ are assumed to have probability density function $f(t)$ and reliability function $R(t)$. The exact lifetime $T_{i}$ of an individual will be observed only if $T_{i} \leq L_{i}$. The lifetime data involving right censoring can be 
conveniently represented by $n$ pairs of random variables $\left(t_{i}, v_{i}\right)$, where $t_{i}=\min \left(T_{i}, L_{i}\right)$ and $v_{i}=1$ if $T_{i} \leq L_{i}$ and $v_{i}=0$ if $T_{i}>L_{i}$. That is, $v_{i}$ indicates whether the lifetime $T_{i}$ is censored or not. The likelihood function is deduced as

$$
L(t)=\prod_{i=1}^{n}\left[f\left(t_{i}\right)\right]^{v_{i}} R\left(t_{i}\right)^{1-v_{i}}
$$

\subsection{Bayesian Modelling for TTF}

Suppose the Time to Failure (TTF) data $t=\left(t_{1}, t_{2}, \cdots, t_{n}\right)^{\prime}$ for $n$ individuals are i.i.d., and each corresponds to a twoparameter Weibull distribution $W(\alpha, \gamma)$, where $\alpha>0$ and $\gamma>0$. Then, the $p . d . f$. is $f\left(t_{i} \mid \alpha, \gamma\right)=\alpha \gamma t_{i}^{\alpha-1} \exp \left(-\gamma t_{i}^{\alpha}\right)$, while the c.d.f. is $F\left(t_{i} \mid \alpha, \gamma\right)=1-\exp \left(-\gamma t_{i}^{\alpha}\right)$, and the reliability function is $R\left(t_{i} \mid \alpha, \gamma\right)=\exp \left(-\gamma t_{i}^{\alpha}\right)$.

Let $v=\left(v_{1}, v_{2}, \cdots, v_{n}\right)^{\prime}$ indicate whether the lifetime is right-censored or not, and let the observed dataset for the study be denoted as $D_{0}$, where $D_{0}=(n, t, v)$, following Equation (3). Therefore, the likelihood function for $\alpha$ and $\gamma$ is

$$
L\left(\alpha, \gamma \mid D_{0}\right)=\alpha^{\sum_{i=1}^{n} v_{i}} \exp \left\{\sum_{i=1}^{n} v_{i} \ln (\gamma)+\sum_{i=1}^{n} v_{i}\left[(\alpha-1) \ln \left(t_{i}\right)-\gamma t_{i}^{\alpha}\right]\right\}
$$

In this study, we take $\alpha$ and $\gamma$ to be independent. Furthermore, we assume $\alpha$ to be a gamma distribution, denoted by $G\left(a_{0}, b_{0}\right)$ as its prior distribution, written as $\pi\left(\alpha \mid a_{0}, b_{0}\right)$, and we assume $\gamma$ to be a gamma distribution denoted by $G\left(c_{0}, d_{0}\right)$ as its prior distribution, written as $\pi\left(\gamma \mid c_{0}, d_{0}\right)$. This means

$$
\begin{aligned}
& \pi\left(\alpha \mid a_{0}, b_{0}\right) \propto \alpha^{a_{0}-1} \exp \left(-b_{0} \alpha\right) \\
& \pi\left(\gamma \mid c_{0}, d_{0}\right) \propto \gamma^{c_{0}-1} \exp \left(-d_{0} \gamma\right)
\end{aligned}
$$

Therefore, the joint posterior distribution can be obtained according to Equations (4) to (6) as

$$
\pi\left(\alpha, \gamma \mid D_{0}\right) \propto L\left(\alpha, \gamma \mid D_{0}\right) \times \pi\left(\alpha \mid a_{0}, b_{0}\right) \times \pi\left(\gamma \mid c_{0}, d_{0}\right)
$$

The parameters' full conditional distribution with Gibbs sampling can be written as

$$
\begin{aligned}
& \pi\left(\alpha_{j} \mid \alpha^{(-j)}, \gamma, D_{0}\right) \propto L\left(\alpha, \gamma \mid D_{0}\right) \times \alpha^{a_{0}-1} \exp \left(-b_{0} \alpha\right) \\
& \pi\left(\gamma_{j} \mid \alpha, \gamma^{(-j)}, D_{0}\right) \propto L\left(\alpha, \gamma \mid D_{0}\right) \times \gamma^{c_{0}-1} \exp \left(-d_{0} \gamma\right)
\end{aligned}
$$

\subsection{Bayesian Modelling for TTR}

Suppose the Time to Repair (TTR) data $t=\left(t_{1}, t_{2}, \cdots, t_{n}\right)^{\prime}$ for $n$ individuals are i.i.d., and each $\ln (t)$ corresponds to a normal distribution $N\left(\mu, \sigma^{2}\right)$. We can obtain $t_{i}$ 's lognormal distribution with parameters $\mu$ and $\sigma^{2}$, denoted by $L N\left(\mu, \sigma^{2}\right)$. Then, the p.d.f. and c.d.f. are given by Equations (10) and (11):

$$
\begin{gathered}
f\left(t_{i} \mid \mu, \sigma^{2}\right)=\frac{1}{\sqrt{2 \pi} \sigma t_{i}} \exp \left\{-\frac{1}{2 \sigma^{2}}\left[\ln \left(t_{i}\right)-\mu\right]^{2}\right\} \\
F\left(t_{i} \mid \mu, \sigma^{2}\right)=\Phi\left[\frac{\ln \left(t_{i}\right)-\mu}{\sigma}\right]
\end{gathered}
$$


The likelihood function related to $\mu$ and $\sigma$, considering the censoring indicators $v=\left(v_{1}, v_{2}, \cdots, v_{n}\right)^{\prime}$ and the observed dataset $D_{0}=(n, t, v)$, becomes

$$
L\left(\mu, \sigma \mid D_{0}\right)=\left(2 \pi \sigma^{2}\right)^{-\frac{1}{2} \sum_{i=1}^{n} v_{i}} \exp \left\{-\frac{1}{2 \sigma^{2}}\left[\ln \left(t_{i}\right)-\mu\right]^{2}\right\} \times \prod_{i=1}^{n} t^{-v_{i}}\left\{1-\Phi\left[\frac{\ln \left(t_{i}\right)-\mu}{\sigma}\right]\right\}^{1-v_{i}}
$$

In this study, we assume $\mu$ to be a normal distribution denoted by $N\left(e_{0}, f_{0}\right)$ as its prior distribution, written as $\pi\left(\mu \mid e_{0}, f_{0}\right)$, and we assume $\sigma$ to be a gamma distribution denoted by $G\left(g_{0}, h_{0}\right)$ as its prior distribution, written as $\pi\left(\sigma \mid g_{0}, h_{0}\right)$. This means

$$
\begin{gathered}
\pi\left(\mu \mid e_{0}, f_{0}\right) \propto f_{0}^{\frac{1}{2}} \exp \left[-\frac{f_{0}}{2}\left(\mu-e_{0}\right)^{2}\right] \\
\pi\left(\sigma \mid g_{0}, h_{0}\right) \propto \sigma^{g_{0}-1} \exp \left(-h_{0} \sigma\right)
\end{gathered}
$$

Therefore, the joint posterior distribution can be obtained according to Equations (12) to (14) as

$$
\pi\left(\mu, \sigma \mid D_{0}\right) \propto L\left(\mu, \sigma \mid D_{0}\right) \times \pi\left(\mu \mid e_{0}, f_{0}\right) \times \pi\left(\sigma \mid g_{0}, h_{0}\right)
$$

The parameters' full conditional distribution with Gibbs sampling can be written as

$$
\begin{gathered}
\pi\left(\mu_{j} \mid \mu^{(-j)}, \sigma, D_{0}\right) \propto L\left(\mu, \sigma \mid D_{0}\right) \times f_{0}^{\frac{1}{2}} \exp \left[-\frac{f_{0}}{2}\left(\mu-e_{0}\right)^{2}\right] \\
\pi\left(\sigma_{j} \mid \mu, \sigma^{(-j)}, D_{0}\right) \propto L\left(\mu, \sigma \mid D_{0}\right) \times \sigma^{g_{0}-1} \exp \left(-h_{0} \sigma\right)
\end{gathered}
$$

\section{Stage III: Assessment}

According to the results from Stage II, the distribution for TTF and TTR can be achieved separately for balling drums 1 to 5 . Compared with the traditional method of assessing availability in Equation (1), the proposed approach extends the method to Equation (18), where

$$
A=\frac{E[f(T T F)]}{E[f(T T F)]+E[f(T T R)]}=\frac{E\left[f\left(t_{i} \mid \alpha, \gamma\right)\right]}{E\left[f\left(t_{i} \mid \alpha, \gamma\right)\right]+E\left[f\left(t_{i} \mid \mu, \sigma^{2}\right)\right]}
$$

Equation (18) shows the flexibility of assessing availability according to reality. The parametric Bayesian models that use MCMC make the calculation of posteriors more feasible.

Based on the system configuration determined in Stage I, and using the results from Stage II for $W(\alpha, \gamma), L N\left(\mu, \sigma^{2}\right)$ and Equation (18), TTF, TTR, and system availability can be assessed.

System availability can be computed via the $T T F$ and TTR. However, according to Equation (18), we cannot obtain a closed-form distribution of system availability. Therefore, we use an empirical distribution instead of an analytical one. As illustrated in the case study, the Kaplan-Meier estimate can be used as the empirical c.d.f.

\section{Case Study}

In this case study of five balling drums, the Markov chain is constructed for each MCMC simulation. A burn-in of 1,000 samples is used, with an additional 10,000 Gibbs samples for each Markov chain. Vague prior distributions are adopted as follows:

- For the Bayesian Weibull model using TTF data: 


$$
\alpha \sim G(0.0001,0.0001), \gamma \sim G(0.0001,0.0001)
$$

- For the Bayesian lognormal model using TTR data:

$$
\mu \sim N(0,0.0001), \sigma \sim G(0.0001,0.0001)
$$

\subsection{Results}

Using convergence diagnostics (i.e., checking dynamic traces in Markov chains, determining time series and GelmanRubin-Brooks (GRB) statistics, and comparing MC error with standard deviation (SD)) [14], we consider the posterior distribution statistics shown in Tables 3 and 4, including the parameters' posterior distribution mean, SD, Monte Carlo error (MC error), and 95\% highest posterior distribution density (HPD) interval.

Table 3. Posterior statistics in Bayesian Weibull model with censored TTF data

\begin{tabular}{|c|c|c|c|c|c|}
\hline Balling drum & Parameter & Mean & SD & MC error & $95 \%$ HPD interval \\
\hline \multirow{2}{*}{1} & $\alpha$ & 0.5399 & 0.0235 & $4.34 \mathrm{E}-4$ & $(0.4954,0.5870)$ \\
\cline { 2 - 5 } & $\gamma$ & 0.0934 & 0.0122 & $2.26 \mathrm{E}-4$ & $(0.0710,0.1186)$ \\
\hline \multirow{2}{*}{2} & $\alpha$ & 0.5721 & 0.0289 & $6.25 \mathrm{E}-4$ & $(0.5159,0.6295)$ \\
\cline { 2 - 5 } & $\gamma$ & 0.0651 & 0.0110 & $2.39 \mathrm{E}-4$ & $(0.0459,0.0890)$ \\
\hline \multirow{2}{*}{3} & $\alpha$ & 0.5781 & 0.0251 & $5.08 \mathrm{E}-4$ & $(0.5299,0.6281)$ \\
\cline { 2 - 5 } & $\gamma$ & 0.0742 & 0.0104 & $2.09 \mathrm{E}-4$ & $(0.0555,0.0961)$ \\
\hline \multirow{2}{*}{4} & $\gamma$ & 0.5713 & 0.0252 & $5.14 \mathrm{E}-4$ & $(0.5228,0.6210)$ \\
\hline \multirow{2}{*}{5} & $\alpha$ & 0.0763 & 0.0109 & $2.22 \mathrm{E}-4$ & $(0.0569,0.0992)$ \\
\cline { 2 - 5 } & $\gamma$ & 0.5601 & 0.0219 & $3.95 \mathrm{E}-4$ & $(0.5176,0.6038)$ \\
\hline
\end{tabular}

Table 4. Posterior statistics in Bayesian lognormal model with censored TTR data

\begin{tabular}{|c|c|c|c|c|c|}
\hline Balling drum & Parameter & Mean & SD & MC error & $95 \%$ HPD interval \\
\hline \multirow{2}{*}{1} & $\mu$ & -0.4501 & 0.0882 & $4.98 \mathrm{E}-4$ & $(-0.6250,-0.2776)$ \\
\cline { 2 - 5 } & $\sigma$ & 0.3585 & 0.0267 & $1.50 \mathrm{E}-4$ & $(0.3078,0.4125)$ \\
\hline \multirow{2}{*}{2} & $\mu$ & -0.3825 & 0.1082 & $6.24 \mathrm{E}-4$ & $(-0.5959,-0.1719)$ \\
\cline { 2 - 5 } & $\sigma$ & 0.3277 & 0.0285 & $1.56 \mathrm{E}-4$ & $(0.2742,0.3853)$ \\
\hline \multirow{2}{*}{3} & $\mu$ & -0.4510 & 0.0839 & $5.10 \mathrm{E}-4$ & $(-0.6176,-0.2871)$ \\
\cline { 2 - 5 } & $\sigma$ & 0.4041 & 0.0305 & $1.80 \mathrm{E}-4$ & $(0.3463,0.4660)$ \\
\cline { 2 - 5 } & $\mu$ & -0.6124 & 0.0907 & $5.29 \mathrm{E}-4$ & $(-0.7924,-0.4351)$ \\
\hline \multirow{2}{*}{4} & $\sigma$ & 0.3516 & 0.0266 & $1.49 \mathrm{E}-4$ & $(0.3010,0.4057)$ \\
\cline { 2 - 5 } & $\mu$ & -0.6023 & 0.0812 & $4.72 \mathrm{E}-4$ & $(-0.7633,-0.4432)$ \\
\hline \multirow{2}{*}{5} & $\sigma$ & 0.3524 & 0.0238 & $1.39 \mathrm{E}-4$ & $(0.3072,0.4007)$ \\
\hline
\end{tabular}

\subsection{Assessment}

Using the results from Tables 3 and 4 for balling drums 1 to 5, we derive the distributions of TTF and TTR as shown in Table 5.

Table 5. Statistics of individual balling drums with censored data

\begin{tabular}{|c|c|c|c|}
\hline \multirow{2}{*}{ Balling drum } & $T T F$ & $T T R$ & Availability \\
\cline { 2 - 4 } & $W(\alpha, \gamma)$ & $L N\left(\mu, \sigma^{2}\right)$ & $1 /\left[1+L N\left(\mu, \sigma^{2}\right) / W(\alpha, \gamma)\right]$ \\
\hline 1 & $W_{1}(0.5399,0.0934)$ & $L N_{1}\left(-0.4501,0.3585^{2}\right)$ & $1 /\left[1+L N_{1}\left(\mu, \sigma^{2}\right) / W_{1}(\alpha, \gamma)\right]$ \\
\hline 2 & $W_{2}(0.5721,0.0651)$ & $L N_{2}\left(-0.3825,0.3277^{2}\right)$ & $1 /\left[1+L N_{2}\left(\mu, \sigma^{2}\right) / W_{2}(\alpha, \gamma)\right]$ \\
\hline 3 & $W_{3}(0.5781,0.0742)$ & $L N_{3}\left(-0.4510,0.4041^{2}\right)$ & $1 /\left[1+L N_{3}\left(\mu, \sigma^{2}\right) / W_{3}(\alpha, \gamma)\right]$ \\
\hline 4 & $W_{4}(0.5713,0.0763)$ & $L N_{4}\left(-0.6124,0.3516^{2}\right)$ & $1 /\left[1+L N_{4}\left(\mu, \sigma^{2}\right) / W_{4}(\alpha, \gamma)\right]$ \\
\hline 5 & $W_{5}(0.5601,0.0940)$ & $L N_{5}\left(-0.6023,0.3524^{2}\right)$ & $1 /\left[1+L N_{5}\left(\mu, \sigma^{2}\right) / W_{5}(\alpha, \gamma)\right]$ \\
\hline
\end{tabular}

Using the results in Table 5, we create p.d.f. and c.d.f. charts of TTF and TTR data in Figures 5 and 6, respectively.

As discussed above, system availability can be computed via the TTF and TTR, but we cannot obtain a closed-form 
distribution of system availability. Therefore, we use an empirical distribution instead of an analytical one. We generate 10,000 samples from the distributions of $T T F$ and $T T R$ and calculate the associated availability. Figure 7 presents the histogram of availability of the five balling drums. We use the Kaplan-Meier estimate as the empirical c.d.f. Figure 8 shows the empirical distribution of the availability of the five balling drums.

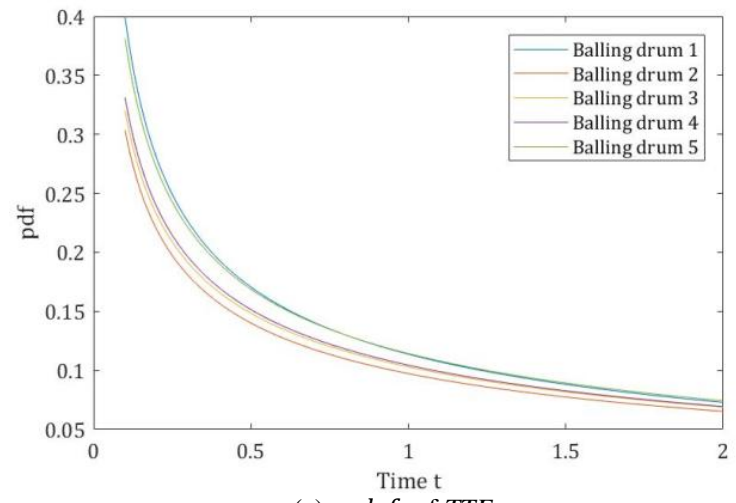

(a) p.d.f. of $T T F$

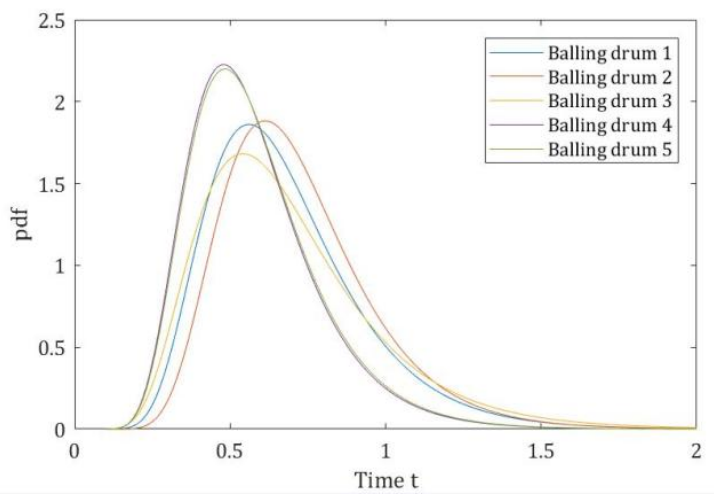

(b) p.d.f. of TTR

Figure 5.p.d.f. of TTF and TTR

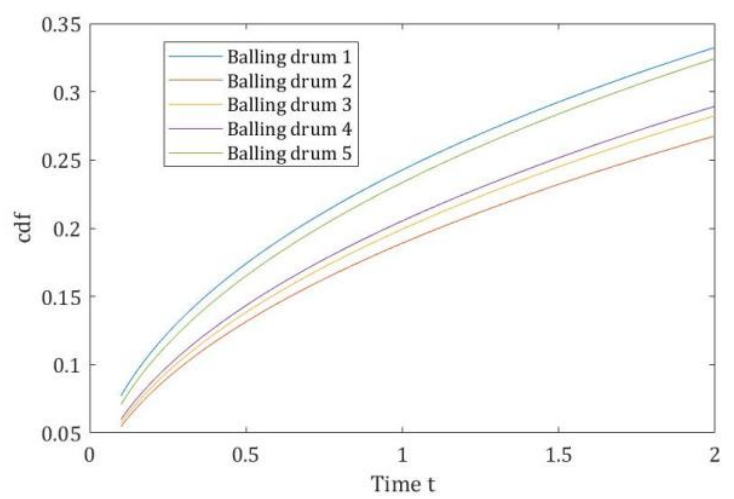

(a) c.d.f. of TTF

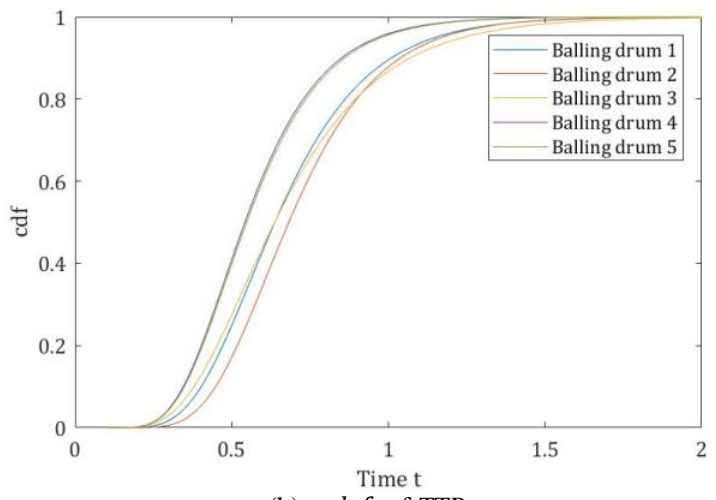

(b) c.d.f. of TTR

Figure 6.c.d.f. of TTF and TTR
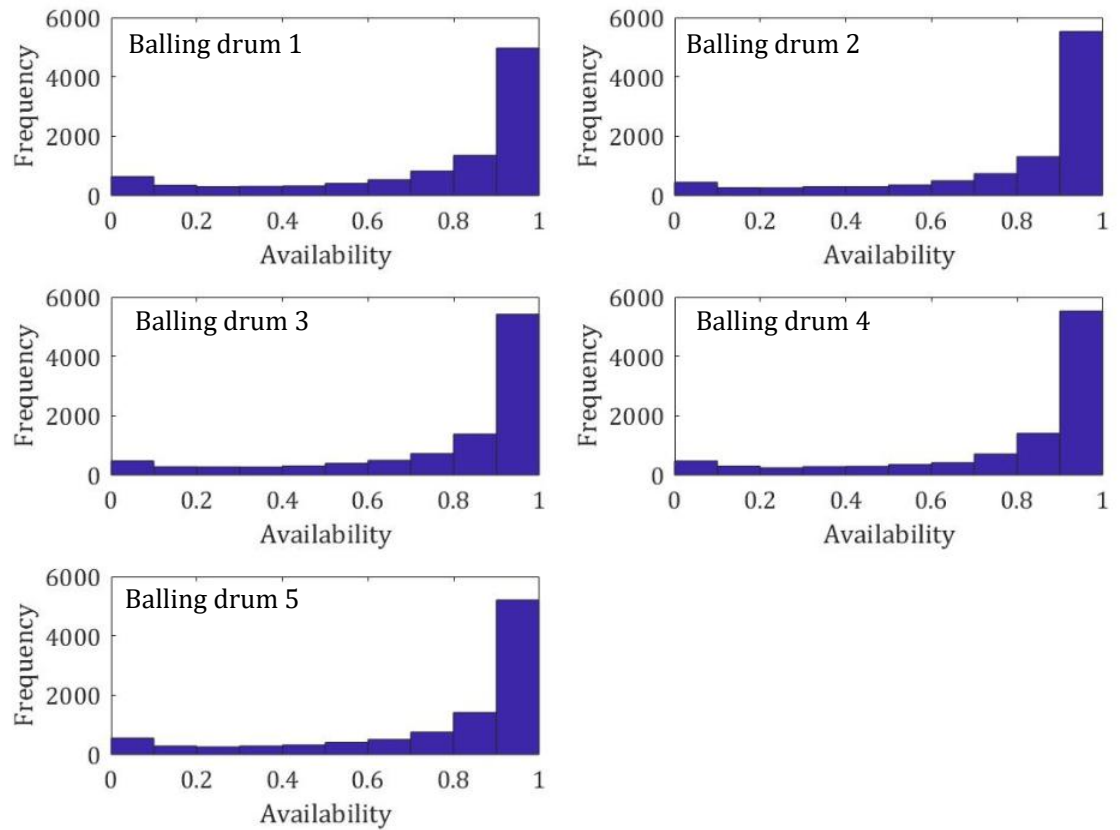

Figure 7. Histogram plot of availability 


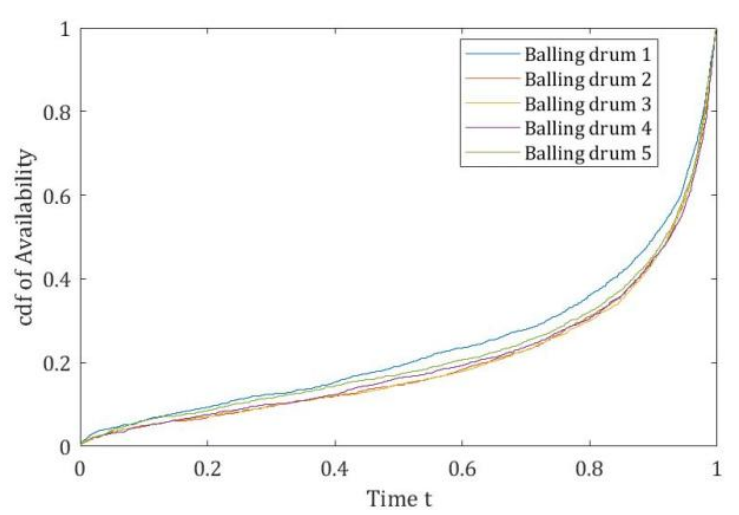

Figure 8. Empirical c.d.f. of availability

We calculate the availability of the individual balling drums in Table 6, where MTTF $=E\left[f\left(t_{i} \mid \alpha, \gamma\right)\right]$, and MTTR $=$ $E\left[f\left(t_{i} \mid \mu, \sigma^{2}\right)\right]$. According to Equation (2), the system availability of the five balling drums is

$$
A_{\text {system }}=1-\prod_{i=1}^{5}\left(1-A_{i}\right) \approx 0.99
$$

Table 6. Statistics of individual balling drums with censored data

\begin{tabular}{|c|c|c|c|c|c|c|}
\hline \multirow{2}{*}{ Balling drum } & \multicolumn{2}{|r|}{ MTTF } & \multicolumn{2}{|r|}{ MTTR } & \multicolumn{2}{|c|}{ Availability } \\
\hline & Mean & 95\% HPD interval & Mean & 95\% HPD interval & Mean & 95\% HPD interval \\
\hline 1 & 145.0 & $(118.4,178.2)$ & 2.616 & $(2.000,3.437)$ & 0.9821 & $(0.9753,0.9873)$ \\
\hline 2 & 197.0 & $(157.6,247.5)$ & 3.223 & $(2.301,4.540)$ & 0.9837 & $(0.9759,0.9893)$ \\
\hline 3 & 146.0 & $(120.7,177.0)$ & 2.239 & $(1.741,2.864)$ & 0.9848 & $(0.9795,0.9890)$ \\
\hline 4 & 149.0 & $(122.5,181.8)$ & 2.289 & $(1.736,3.041)$ & 0.9847 & $(0.9788,0.9891)$ \\
\hline 5 & 115.0 & $(96.40,137.5)$ & 2.296 & $(1.796,2.958)$ & 0.9803 & $(0.9736,0.9855)$ \\
\hline
\end{tabular}

\section{Discussion}

\subsection{A Comparison Study}

For comparative purposes, Tables 7 and 8 show the statistics of the individual balling drums with no censored data. All TTF and TTR data collected in Stage I are treated as reasonable and require no improvement.

Table 7. Statistics of individual balling drums with no censored data

\begin{tabular}{|c|c|c|c|}
\hline \multirow{2}{*}{ Balling drum } & $T T F$ & $T T R$ & Availability \\
\cline { 2 - 4 } & $W(\alpha, \gamma)$ & $L N\left(\mu, \sigma^{2}\right)$ & $1 /\left[1+L N\left(\mu, \sigma^{2}\right) / W(\alpha, \gamma)\right]$ \\
\hline 1 & $W_{1}(0.5409,0.0928)$ & $L N_{1}\left(-0.1842,0.2270^{2}\right)$ & $1 /\left[1+L N_{1}\left(\mu, \sigma^{2}\right) / W_{1}(\alpha, \gamma)\right]$ \\
\hline 2 & $W_{2}(0.5747,0.0642)$ & $L N_{2}\left(-0.0075,0.1861^{2}\right)$ & $1 /\left[1+L N_{2}\left(\mu, \sigma^{2}\right) / W_{2}(\alpha, \gamma)\right]$ \\
\hline 3 & $W_{3}(0.5975,0.0712)$ & $L N_{3}\left(-0.4574,0.2196^{2}\right)$ & $1 /\left[1+L N_{3}\left(\mu, \sigma^{2}\right) / W_{3}(\alpha, \gamma)\right]$ \\
\hline 4 & $W_{4}(0.5745,0.0750)$ & $L N_{4}\left(-0.3540,0.2184^{2}\right)$ & $1 /\left[1+L N_{4}\left(\mu, \sigma^{2}\right) / W_{4}(\alpha, \gamma)\right]$ \\
\hline 5 & $W_{5}(0.5660,0.0958)$ & $L N_{5}\left(-0.3484,0.2195^{2}\right)$ & $1 /\left[1+L N_{5}\left(\mu, \sigma^{2}\right) / W_{5}(\alpha, \gamma)\right]$ \\
\hline
\end{tabular}

Table 8. Statistics of individual balling drums with no censored data

\begin{tabular}{|c|c|c|c|c|c|c|}
\hline \multirow{2}{*}{ Balling drum } & \multicolumn{2}{|c|}{$M T T F$} & \multicolumn{2}{|c|}{ MTTR } & \multicolumn{2}{|c|}{ Availability } \\
\cline { 2 - 6 } & Mean & 95\% HPD interval & Mean & 95\% HPD interval & Mean & 95\% HPD interval \\
\hline 1 & 145.0 & $(118.1,178.0)$ & 7.779 & $(5.284,11.58)$ & 0.9487 & $(0.9229,0.9665)$ \\
\hline 2 & 196.4 & $(157.7,256.0)$ & 15.48 & $(8.927,26.60)$ & 0.9265 & $(0.8766,0.9582)$ \\
\hline 3 & 128.7 & $(127.9,155.0)$ & 6.381 & $(6.194,9.622)$ & 0.9525 & $(0.9538,0.9693)$ \\
\hline 4 & 148.5 & $(122.5,180.3)$ & 7.178 & $(4.755,10.86)$ & 0.9536 & $(0.9291,0.9702)$ \\
\hline 5 & 115.8 & $(115.1,139.0)$ & 7.083 & $(6.926,10.22)$ & 0.9420 & $(0.9433,0.9610)$ \\
\hline
\end{tabular}

For convenience, the results are also listed in Table 9. 
Table 9. Comparison of statistics with and without censored data

\begin{tabular}{|c|c|c|c|c|c|c|c|c|c|}
\hline \multirow{2}{*}{$\begin{array}{l}\text { Balling } \\
\text { drum }\end{array}$} & \multicolumn{3}{|c|}{ Mean of $M T T F$} & \multicolumn{3}{|c|}{ Mean of $M T T R$} & \multicolumn{3}{|c|}{ Mean of Availability } \\
\hline & No censored & Censored & $\%$ & No censored & Censored & $\%$ & No censored & Censored & $\%$ \\
\hline 1 & 145.0 & 145.0 & 0 & 7.779 & 2.616 & 66.37 & 0.9487 & 0.9821 & 3.52 \\
\hline 2 & 196.4 & 197.0 & 0.30 & 15.48 & 3.223 & 79.18 & 0.9265 & 0.9837 & 6.17 \\
\hline 3 & 128.7 & 146.0 & 13.4 & 6.381 & 2.239 & 64.91 & 0.9525 & 0.9848 & 3.39 \\
\hline 4 & 148.5 & 149.0 & 0.33 & 7.178 & 2.289 & 68.11 & 0.9536 & 0.9847 & 3.26 \\
\hline 5 & 115.8 & 115.0 & 0 & 7.083 & 2.296 & 67.58 & 0.9420 & 0.9803 & 4.07 \\
\hline
\end{tabular}

In Table 9, "\%" denotes the percentage after considering the censored data. For instance, for balling drum 1, after considering the censored data, the mean of MTTF does not change; MTTR improves by $66.37 \%$, and the availability improves by $3.52 \%$.

According to the results from Table 9, if $20 \%$ of the abnormal TTR data could be improved (for instance, by applying RCA activities, or more specifically, by improving maintenance resource management, including maintenance skills, spare parts, etc.), the TTR could be improved by $66.37 \%, 79.18 \%, 64.91 \%, 68.11 \%$, and $67.58 \%$ for drums 1 to 5 , respectively. Meanwhile, the availability would be improved by $3.52 \%, 6.17 \%, 3.39 \%, 3.26 \%$, and $4.07 \%$ for drums 1 to 5 , respectively.

The improvement of the $T T F$ is not as impressive. We apply right-censored data for the TTRs under the assumption that they can be improved (censored at six), but the corresponding TTFs can only be marked as censored instead of censored at some specified value, under the assumption that the maintenance interval will not change that much. This implies that if the maintenance interval (for instance, the preventive maintenance) could be improved, the TTFs could be improved (censored at a larger value), thus improving the availability.

\subsection{Connection Between Technical and "Soft" KPIs}

In the studied company, Key Performance Indicators (KPIs) are divided into two groups: technical KPIs and soft KPIs. The former is related to the performance of equipment, while the latter focuses on maintenance management.

In this case, the abnormal values of TTR are assumed to be mainly caused by a lack of maintenance resources, including personnel with suitable skills, spare parts, etc. KPIs of maintenance resources are treated as "soft" KPIs in the company. Therefore, using our comparative approach, we could easily determine how the technical KPIs (TTF, availability of assets) would be influenced by improving "soft" KPIs.

\subsection{Application of the Threshold as a Monitoring Line}

In this study, the threshold of abnormal TTR values in the work orders is determined by a "80-20" rule in Pareto analysis, in which a $T T R$ value exceeding six is treated as an abnormally long time for TTR and should be improved by RCA activities, including improving maintenance resource management.

Actually, the threshold could be determined by the company according to its business goals; for instance, they could be set at $70 \%$ or $90 \%$, or set according to other rules combined with business goals. The threshold could also be changed gradually to improve the maintenance step by step, following a PDCA process. In another words, the so-called abnormal data are not really abnormal. Finally, the threshold could be treated as a monitoring line, permitting the dynamic monitoring of system availability.

\subsection{Further Research}

In this study, since the five balling drums are relatively new, the gamma distributions and normal distributions are selected as vague priors due to lack of real prior information. This could be improved with more historical data/experience.

The system configurations could be extended to other more complex architectures (series, k-out-of-n, stand-by, multistate, or mixed) by modifying Equation (2).

The results of system availability are all larger than 0.99 , with or without considering censored data. The difference is not very obvious for two reasons. First, the system configuration is in parallel; second, the individual balling drums have 
relatively high availabilities (higher than 0.9 ). The difference (with or without considering censored data) will be more obvious with other system configurations and less individual availability.

For TTF data, the shape parameter for the Weibull distribution is less than 1 (see Figure 5 (a)). The TTFs have a decreasing trend (as in the early stage of the bathtub curve), which is not suitable for the real-world experience of mechanical equipment. However, the TTF data include not only corrective maintenance but also preventive maintenance. The decreasing trends suggest that a possible way to improve $T T F$ is to improve the preventive maintenance plan.

\section{Conclusions}

This study proposes a parametric Bayesian approach to assess system availability in the operational stage. MCMC is adopted to take advantage of both analytical and simulation methods. Because of MCMC's high dimensional numerical integral calculation, the selection of prior information and descriptions of reliability/maintainability can be more flexible and realistic. In this method, MTTF and MTTR are treated as distributions instead of being "averaged" by point estimation. This better reflects reality; in addition, the limitations of simulation data sample size are overcome by MCMC techniques.

In the case study, TTF and TTR are determined using a Bayesian Weibull model and a Bayesian lognormal model, respectively. The results show the following:

- The proposed approach can integrate analytical and simulation methods for system availability assessment and could be applied to other technical problems in asset management (e.g., other industries, other systems);

- There is a connection between technical and "soft" KPIs;

- The threshold can be treated as a monitoring line by the mining company for continuous improvement.

\section{Acknowledgments}

The motivation for the research was the project "Key Performance Indicators (KPIs) for control and management of maintenance process through eMaintenance" (in Swedish: Nyckeltal för styrning och uppföljning av underhållsverksamhet $\mathrm{m} \mathrm{h}$ a eUnderhåll), which was initiated and financed by LKAB. The authors would like to thank Ramin Karim, Peter Olofsson, Mats Renfors, Sylvia Simma, Maria Rytty, Mikael From, and Johan Enbak for their support of this research in the form of funding and work hours.

\section{References}

1. D. Raje, R. Olaniya, P. Wakhare, and A. Deshpande, "Availability Assessment of a Two-unit Stand-by Pumping System," Reliability Engineering and System Safety, Vol. 68, pp. 269-274, 2000

2. A. C. Marquez, A. S. Heguedas, and B. Iung, "Monte Carlo-based Assessment of System Availability. A Case Study for Cogeneration Plants," Reliability Engineering and System Safety, Vol. 88, pp. 273-289, 2005

3. A. C. Marquez and B. Iung, "A Structured Approach for the Assessment of System Availability and Reliability using Monte Carlo Simulation," Journal of Quality in Maintenance Engineering, Vol. 13, No. 2, pp. 125-136, 2007

4. S. F. Yasseri and H. Bahai, "Availability Assessment of Subsea Distribution Systems at the Architectural Level," Ocean Engineering, Vol. 153, pp. 399-411, 2018

5. E. Zio, M. Marella, and L. podofillini, "A Monte Carlo Simulation Approach to the Availability Assessment of Multi-State System with Operational Dependencies," Reliability Engineering and System Safety, Vol. 92, pp. 871-882, 2007

6. R. Dekker and W. Groenendijk, "Availability Assessment Methods and their Application in Practice," Microelectronics Reliability, Vol. 35, No. 9-10, pp. 1257-1274, 1995

7. A. B. Ocnasu, "Distribution System Availability Assessment-Monte Carlo and Antithetic Variates Method," Vienna, 2007

8. S. Faghih-Roohi, M. Xie, K. M. Ng, and R. C. Yam, "Dynamic Availability Assessment and Optimal Component Design of Multi-state Weighted k-out-of-n Systems," Reliability Engineering and System Safety, Vol. 123, pp. 57-62, 2014

9. D. M. Brender, "The Bayesian Assessment of System Availability: Advanced Applications and Techniques," IEEE Transactions on Reliability, Vol. 17, No. 3, pp. 138-147, 1968

10. D. M. Brender, "The Prediction and Measurement of System Availability: A Bayesian Treatment," IEEE Transactions on Reliability, Vol. 17, No. 3, pp. 127-138, 1968

11. W. Kuo, "Bayesian Availability using Gamma Distributed Priors," IIE Transactions, Vol. 17, No. 2, pp. 132-140, 1985

12. K. Sharma and R. Bhutani, "Bayesian Analysis of System Availability," Microelectronic Reliability, Vol. 33, No. 6, pp. 809811,1993

13. M. A. Khan and H. Islam, "Bayesian Analysis of System Availability with Half-Normal Life Time," Quality Technology and Quantitative Management, Vol. 9, No. 2, pp. 203-209, 2012

14. J. Lin, "An Integrated Procedure for Bayesian Reliability Inference using Markov Chain Monte Carlo Methods," Journal of Quality and Reliability Engineering, Vol. 2014, pp. 1-16, 2014 